\title{
Effects of a 15-month anti-TNF-a treatment on plasma levels of glycosaminoglycans in women with rheumatoid arthritis
}

\author{
Anna Szeremeta ${ }^{1 *}$, Agnieszka Jura-Półtorak', Ewa Maria Koźma', Andrzej Głowacki', Eugeniusz Józef Kucharz², \\ Magdalena Kopeć-Mędrek² and Krystyna Olczyk ${ }^{1}$
}

\begin{abstract}
Background: In this study, the effect of 15-month anti-tumor necrosis factor alpha (TNF-a) treatment on circulating levels of plasma sulfated glycosaminoglycans (GAGs) and the nonsulfated GAG hyaluronic acid (HA) in female rheumatoid arthritis (RA) patients was assessed.

Methods: Plasma was obtained from healthy subjects and RA women treated with TNF-a antagonists (etanercept or adalimumab or certolizumab pegol) in combination with methotrexate. GAGs were isolated from plasma samples using ion exchange low-pressure liquid chromatography. Total sulfated GAGs were quantified using a hexuronic acid assay. Plasma levels of keratan sulfate (KS) and HA were measured using immunoassay kits.
\end{abstract}

Results: Total sulfated GAGs and HA levels were higher in female RA patients before treatment in comparison to healthy subjects. KS levels did not differ between RA women and controls. Anti-TNF-a treatment resulted in normalization of plasma total GAG and HA levels in RA patients, without any effect on KS levels.

Conclusions: Our results suggest that anti-TNF-a therapy has a beneficial effect on extracellular matrix remodeling in the course of RA.

Keywords: Rheumatoid arthritis, Tumor necrosis factor-alpha inhibitors, Glycosaminoglycans, Keratan sulfate, Hyaluronic acid

\section{Background}

Rheumatoid arthritis (RA) is a chronic, systemic, autoimmune connective tissue disease characterized by nonspecific arthritis of symmetric joints, as well as progressive articular cartilage degradation and bone erosion [1]. In the course of the disease there are numerous extra-articular organ manifestations which are the major causes of fast-growing patient disability. RA is associated with a higher mortality rate compared to that of the general population. RA affects approximately $0.5-1.5 \%$ of the world's population at any age. The incidence peaks in the $40-50$ age group and women suffer from RA nearly two or three times more frequently than men [2-4].

\footnotetext{
* Correspondence: aszeremeta@sum.edu.pl

${ }^{1}$ Department of Clinical Chemistry and Laboratory Diagnostics, School of Pharmacy with the Division of Laboratory Medicine in Sosnowiec, Medical University of Silesia in Katowice, Jedności 8, 41-200 Sosnowiec, Poland Full list of author information is available at the end of the article
}

Despite many studies, the causes of RA are still not completely known [3-5]. Tumor necrosis factor alpha $(\mathrm{TNF}-\alpha)$ is one of the main proinflammatory cytokines, playing a key role in the pathogenesis of the disease. It has been shown that TNF- $\alpha$ stimulates catabolic processes in the cartilage tissue and periarticular structures and significantly affects the induction and persistence of inflammation [4-7]. Some of the integral elements of chronic inflammation are the structural and functional changes in extracellular matrix (ECM) compounds, including proteoglycans (PGs) and their sugar constituents-that is, glycosaminoglycans (GAGs) $[8,9]$. The latter are negatively charged unbranched polysaccharides consisting of repeating disaccharide units of hexosamine and uronic acid or galactose. Chondroitin/dermatan sulfate (CS/DS), heparan sulfate/heparin $(\mathrm{HS} / \mathrm{H})$, keratan sulfate (KS), and hyaluronic acid (HA) represent the major species of GAGs. All GAG types, except HA, are

(C) The Author(s). 2018 Open Access This article is distributed under the terms of the Creative Commons Attribution 4.0 International License (http://creativecommons.org/licenses/by/4.0/), which permits unrestricted use, distribution, and 
covalently attached to the core protein, forming PGs, and exhibit various degrees of sulfation along the polysaccharide chain [10-12]. This structural heterogeneity allows GAGs to interact with and modify the actions of numerous cell-adhesion molecules, growth factors, cytokines, chemokines, components of ECM, proteases, and their inhibitors, which underlie their important biological functions, including cellular communication, cell signaling, and regulation of other biochemical pathways $[10,13]$. Hence, every modification of GAG metabolism may play a crucial role in RA pathogenesis. The disturbed balance between biosynthesis and degradation of PGs/GAGs as a consequence of chronic inflammation during RA should be reflected in the concentration of plasma GAGs. Friman et al. [14] suggested that in patients suffering from an active, erosive form of RA, the total plasma GAG content is not different as compared to controls. On the other hand, Jura-Półtorak et al. [15] described an increase in the total plasma GAG level in patients with RA.

The introduction of biologic drugs which neutralize TNF- $\alpha$ activity was a breakthrough in RA treatment. The results of clinical trials revealed a significant reduction of disease activity and inhibition of radiological progression, as well as improvement in the quality of life and physical function in RA patients treated with TNF- $\alpha$ inhibitors (TNFoI) [16-18]. However, the effect of anti-TNF therapy on PG/GAG metabolism in RA is still unknown. Therefore, the main objective of this study was the quantitative evaluation of total plasma sulfated and nonsulfated GAGs in female RA patients, both before and during 15 months of anti-TNF- $\alpha$ therapy.

\section{Methods}

\section{Patients and samples}

Forty-five female patients (mean \pm SD age $47.42 \pm 13.70$ years) meeting the 1987 revised criteria and the 2010 American College of Rheumatology (ACR)/European League Against Rheumatism (EULAR) diagnostic criteria for RA $[19,20]$ were recruited for this study. All subjects had a Disease Activity Score of 28 joints (DAS28) > 5.1 despite application of at least two disease-modifying anti-rheumatic drugs at trial entry. Exclusion criteria included previous treatment with biologic agents, withdrawing from the biologic therapy during the study period, presence of illnesses (other autoimmune diseases, infections, heart failure, diabetes mellitus, thyroid disorders, kidney, and liver disease or malignancies), pregnancy, and breast-feeding. Moreover, all of the female RA patients participated in Polish National Health Fund Therapeutic Programs employing TNF blockersthat is, B.33: "Treatment of aggressive forms of rheumatoid arthritis (RA) and juvenile idiopathic arthritis (JIA)" (03.0000.333.02), or B.45: "Treatment of an aggressive form of rheumatoid arthritis (03.0000.345.02)"-which were valid during 2012-2014. Twenty-two patients received adalimumab $40 \mathrm{mg}$ subcutaneously every other week and 19 patients self-administered etanercept $50 \mathrm{mg}$ by subcutaneous injection once a week, and four patients received certolizumab pegol $400 \mathrm{mg}$ subcutaneously at weeks 0 , 2, and 4 followed by $200 \mathrm{mg}$ every 2 weeks thereafter for a period of 15 months. Patients were continuing current antirheumatic therapy, including methotrexate ( $25 \mathrm{mg} /$ week) and prednisone ( $\leq 7.5 \mathrm{mg} /$ day). All subjects were given folic acid in the dose of $5 \mathrm{mg} /$ day. Concomitant medications remained unchanged for the duration of the study. Baseline characteristics of patients are presented in Table 1.

The effectiveness of TNF $\alpha$ I treatment was assessed at the baseline of the study and 3, 9, and 15 months after starting anti-TNF- $\alpha$ therapy using the DAS28 indicator, calculated on the basis of the number of swollen and tender joints from among the 28 joints included, the erythrocyte sedimentation rate (ESR), and the patient's global assessment of disease activity on a visual analog scale (VAS) of $100 \mathrm{~mm}$. Furthermore, at each visit, patients were submitted to laboratory tests, such as complete blood count, markers of inflammation including the ESR and C-reactive protein (CRP), creatinine, and liver enzymes. Changes in clinical characteristics during the 15-month TNFaI therapy are summarized in Table 2. Patients who did not experience an

Table 1 Baseline characteristics of female RA patients treated with TNFal

\begin{tabular}{ll}
\hline Characteristic & Value \\
\hline All women with RA, $n(\%)$ & $45(100)$ \\
Age (years), mean (SD) & $47.42(13.70)$ \\
Disease duration (years), median (IQR) & $7(4-16)$ \\
BMI (kg/m²), mean (SD) & $21.99(2.26)$ \\
RF positive, $n$ (\%) & $44(97.78)$ \\
Anti-CCP positive, $n(\%)$ & $45(100)$ \\
SJC28, median (IQR) & $8(5-10)$ \\
TJC28, median (IQR) & $14(10-19)$ \\
VAS, median (IQR) & $80(80-80)$ \\
DAS28 ESR, mean (SD) & $6.10(0.58)$ \\
ESR (mm/h), median (IQR) & $18.0(11.0-33.0)$ \\
CRP (mg/l), median (IQR) & $5.0(4.0-14.9)$ \\
TNFal therapy, $n$ (\%) & \\
Etanercept (Enbrel) & $19(42.22)$ \\
Adalimumab (Humira) & $22(48.89)$ \\
Certolizumab pegol (Cimzia) & $4(8.89)$ \\
\hline
\end{tabular}

anti-CCP anti-cyclic citrullinated peptide antibody, BMI body mass index, $C R P$ C-reactive protein, DAS28 Disease Activity Score based on evaluation of 28 joints, ESR erythrocyte sedimentation rate, IQR interquartile range, $R A$ rheumatoid arthritis, $R F$ rheumatoid factor, $S D$ standard deviation, $S J C 28$ swollen joint count of 28 joints, TJC28 tender joint count of 28 joints, TNFal tumor necrosis factor-alpha inhibitors, VAS visual analog scale 
adequate treatment response were excluded from the study. Adequate treatment response in accordance with the principles of the Polish National Health Fund Therapeutic Programs was defined as reduction in DAS28 $>1.2$ after the first 3 months of therapy with a TNF- $\alpha$ inhibitor, and further reduction in DAS28 by 1.2 recorded during subsequent medical examinations performed 9 and 15 months after administration of the first dose of TNF $\alpha$ I.

Twenty age-matched healthy female volunteers from the Medical University of Silesia in Katowice, Poland were investigated as controls. Subjects were selected after their medical history, clinical examination, and laboratory screening had been obtained. All volunteers enrolled in this study did not have any diseases that required hospitalization and did not undergo surgical procedures during the previous 3 years. Furthermore, the results of their routine laboratory tests (i.e., complete blood count, ESR, fasting glucose, fasting lipid profile, creatinine, liver enzymes, rheumatoid factor (RF), and CRP) were within the reference range. Subjects were excluded if they took steroidal or nonsteroidal anti-inflammatory drugs. None of the volunteers smoked cigarettes or had any history of drug or alcohol abuse. We selected women who could maintain a healthy body weight and had a body mass index $(\mathrm{BMI})<25 \mathrm{~kg} / \mathrm{m}^{2}$.

On the day of collecting the plasma, prior to that procedure, patients met with rheumatologists for clinical visits, during which assessment of the patient, the physical visual analog scale of disease (VAS), the tender joint count of 28 joints (TJC28), the swollen joint count of 28 joints (SJC28), and the DAS28 were made. Venous blood samples were drawn between 7.00 and 9.00 am after overnight fasting, and were collected into citrate-treated (extraction and determination of plasma GAGs) and heparin-treated (measurement of plasma KS and HA levels) tubes. Plasma samples obtained from healthy subjects and RA patients were separated and stored at $80{ }^{\circ} \mathrm{C}$ until the time of analysis.

Table 2 Time-course changes in biochemical, clinical, and functional measures during 15-month anti-TNF-a therapy

\begin{tabular}{|c|c|c|c|c|}
\hline & \multicolumn{4}{|c|}{ Time after starting anti-TNF-a therapy } \\
\hline & $\mathrm{T}_{0}$ (baseline) & $\mathrm{T}_{1}$ (3 months) & $\mathrm{T}_{2}$ (9 months) & $\mathrm{T}_{3}$ (15 months) \\
\hline Women with RA, $n(\%)$ & $29(100)$ & & & \\
\hline Age (years), mean (SD) & $44.38(14.17)$ & & & \\
\hline Disease duration (years), median (IQR) & $5(3-8)$ & & & \\
\hline BMI $\left(\mathrm{kg} / \mathrm{m}^{2}\right)$, mean $(\mathrm{SD})$ & $21.25(2.28)$ & & & \\
\hline RF positive, $n(\%)$ & $29(100)$ & & & \\
\hline Anti-CCP positive, $n(\%)$ & $29(100)$ & & & \\
\hline SJC28, median (IQR) & $6(5-10)$ & $3(2-3)^{a, c, d}$ & $0(0-1)^{a, b}$ & $0(0-0)^{a, b}$ \\
\hline TJC28, median (IQR) & $14(10-20)$ & $5(3-7)^{a, c, d}$ & $2(1-2)^{a, b, d}$ & $0(0-1)^{a, b, c}$ \\
\hline VAS, median (IQR) & $80(80-80)$ & $50(35-55)^{a, c, d}$ & $25(10-30)^{a, b, d}$ & $10(5-20)^{a, b}, c$ \\
\hline DAS28 ESR, mean (SD) & $5.99(0.50)$ & $4.00(0.73)^{a, c, d}$ & $2.74(0.72)^{a, b, d}$ & $2.06(0.64)^{a, b, c}$ \\
\hline \multicolumn{5}{|l|}{ Disease activity, $n$ (\%) } \\
\hline High (> 5.1) & $29(100)$ & $2(6.90)$ & 0 & 0 \\
\hline Moderate (> 3.2 and $\leq 5.1$ ) & 0 & $24(82.76)$ & $6(20.69)$ & 0 \\
\hline Low ( $\leq 3.2$ and $>2.6$ ) & 0 & $3(10.34)$ & $12(41.38)$ & $6(20.69)$ \\
\hline Remission $(\leq 2.6)$ & 0 & 0 & $11(37.93)$ & $23(79.31)$ \\
\hline ESR $(\mathrm{mm} / \mathrm{h})$, median (IQR) & $15.0(10.0-31.0)$ & $10.0(8.0-17.0)$ & $10.0(8.0-14.0)^{\mathrm{a}}$ & $11.0(8.0-14.0)^{\mathrm{a}}$ \\
\hline CRP (mg/l), median (IQR) & $5.0(4.0-9.2)$ & $4.0(2.0-4.0)$ & $3.0(1.30-4.0)^{\mathrm{a}}$ & $2.0(1.0-4.0)^{\mathrm{a}}$ \\
\hline \multicolumn{5}{|l|}{ TNFal therapy, $n(\%)$} \\
\hline Etanercept (Enbrel) & $13(44.83)$ & & & \\
\hline Adalimumab (Humira) & $14(48.27)$ & & & \\
\hline Certolizumab pegol (Cimzia) & $2(6.90)$ & & & \\
\hline
\end{tabular}

Differences noted for all variables (except DAS28 ESR) considered significant at $p<0.0083$ by applying Bonferroni correction. Differences noted for DAS28 ESR considered significant at $p<0.001$

anti-CCP anti-cyclic citrullinated peptide antibody, BMI body mass index, CRP C-reactive protein, DAS28 Disease Activity Score based on evaluation of 28 joints, ESR erythrocyte sedimentation rate, IQR interquartile range, $R A$ rheumatoid arthritis, $R F$ rheumatoid factor, $S D$ standard deviation, $S J C 28$ swollen joint count of 28 joints, TJC28 tender joint count of 28 joints, TNF-a tumor necrosis factor alpha, TNFal tumor necrosis factor-alpha inhibitors, VAS Visual analog scale

${ }^{\text {a S}}$ Statistically significant differences compared to $\mathrm{T}_{0}$

${ }^{\mathrm{b}}$ Statistically significant differences compared to $\mathrm{T}_{1}$

'Statistically significant differences compared to $T_{2}$

${ }^{d}$ Statistically significant differences compared to $T_{3}$ 
During the entire investigation period we followed the guidelines and regulations of the Helsinki Declaration in 1975, as revised in 1983. The Ethical Committee of the Medical University of Silesia in Katowice approved the research protocol used in this study. All healthy volunteers and RA patients provided written informed consent.

\section{Extraction and determination of total plasma GAG levels}

Sulfated GAGs were isolated using the methods of $\mathrm{Lu}$ et al. [21] and Capobianco et al. [22]. Firstly, plasma samples $(1 \mathrm{ml})$ were pretreated for $24 \mathrm{~h}$ at $37{ }^{\circ} \mathrm{C}$ with Benzonase (E1014; Sigma) for the removal of nucleic acids. Next, plasma samples were submitted to exhaustive digestion with Streptomyces griseus protease (P0652; Sigma) in order to release GAG chains from plasma PG core proteins. This proteolysis was performed in $20 \mathrm{mM}$ Tris- $\mathrm{HCl}$, pH 9.0, containing $4 \mathrm{mM} \mathrm{CaCl}_{2}$, at $56{ }^{\circ} \mathrm{C}$ for $24 \mathrm{~h}$. After incubation, the samples were centrifuged at $19,000 \times g$ for $30 \mathrm{~min}$ and the pellet was washed twice with the same buffer. The combined supernatants were applied to DEAE-Sephacel columns equilibrated with $0.1 \mathrm{M} \mathrm{NaCl}$ buffered with $20 \mathrm{mM}$ Tris- $\mathrm{HCl}, \mathrm{pH}$ 8.6, and columns were washed with the same buffer. GAGs were eluted with $2 \mathrm{M} \mathrm{LiCl}$ in $20 \mathrm{mM}$ Tris- $\mathrm{HCl}, \mathrm{pH}$ 8.6. Subsequently, GAGs were exhaustively dialyzed against water at $4{ }^{\circ} \mathrm{C}$ for $24 \mathrm{~h}$ and lyophilized before further analysis.

The total amount of GAGs was quantified as a hexuronic acid by the carbazole methods of Volpi et al. [23] and Filisetti-Cozzi and Carpita [24] as well as van den Hoogen et al. [25]. Then, 4.0 M ammonium sulfamate was added to each sample containing an aqueous solution of GAGs. The hydrolysis of GAGs to their monosaccharide constituents with simultaneous conversion of the glucuronic acid and/or iduronic acid residues to the corresponding furan derivative was carried out in concentrated sulfuric acid (95\%) containing $0.025 \mathrm{M}$ sodium tetraborate by heating the samples at $100{ }^{\circ} \mathrm{C}$ for $10 \mathrm{~min}$. The contents of the tubes were then chilled in an ice bath and the background absorbance of the samples was measured at $525 \mathrm{~nm}$ with a microplate reader (Infinite M200; Tecan). In the next stage, furan derivatives present in the tested samples were coupled with $0.125 \%$ carbazole that was dissolved in absolute ethanol. The tubes were heated to $100{ }^{\circ} \mathrm{C}$ for $15 \mathrm{~min}$ and left to cool to ambient temperature. Afterward, the absorbance of the pink-colored samples was read again at $525 \mathrm{~nm}$. For hexuronic acid quantification, a calibration curve was constructed using D-(+) glucuronolactone standard series $(0-70 \mu \mathrm{g} / \mathrm{ml})$. The background absorbance was subtracted from the second absorbance reading and the hexuronic acid concentrations were interpolated from the corresponding reference curve. The sensitivity of the reaction was approximately $1.5 \mu \mathrm{g}$ for glucuronic acid. Testing of all samples was completed in 1 day, so interassay variation was insignificant. The intraassay variability of total GAGs was less than 3\%.

\section{Measurement of KS and HA plasma levels}

$\mathrm{KS}$ levels in plasma samples were measured in duplicate using an enzyme-linked immunosorbent assay (ELISA) from BlueGene Biotech (Shanghai, China) according to the manufacturer's instructions. The minimal detectable $\mathrm{KS}$ level was $0.1 \mathrm{ng} / \mathrm{ml}$. All samples were tested within 1 day, and thus interassay variation was insignificant. The intraassay variation of the KS levels was $<10 \%$.

Plasma concentrations of HA were determined in duplicate using a $\mathrm{TECO}^{\circ}$ Hyaluronic Acid Plus Test Kit provided by TECOmedical Group (Sissach, Switzerland), according to the manufacturer's directions. This ELISA uses a highly specific hyaluronic acid binding protein (HABP) to capture HA and an enzyme-conjugated version of the HABP to detect and measure the HA captured from plasma samples. In brief, all plasma samples were diluted 50-fold with Sample Diluent. The analytical sensitivity was at $2.7 \mathrm{ng} / \mathrm{ml}$. Testing of all samples was completed within 1 day to eliminate the effects of interassay variation. The intraassay coefficient of variation was $<2.9 \%$.

\section{Statistical analysis}

Data analyses were performed using STATISTICA version 12 (https://www.statsoft.pl). The normality of the distribution was verified using the Shapiro-Wilk test. Data not normally distributed were log-transformed prior to analyses. Variables are summarized as mean (SD) (for normal distribution) or median and interquartile (25th-75th percentile) range (for abnormal distribution). The homogeneity of variance was assessed using Levene's test. Data were evaluated using a repeated-measures analysis of variance (RM-ANOVA) (normal distributed data) with a check for sphericity employing Mauchly's test of sphericity, or using the RM-ANOVA Friedman's test (nonnormal data). In the case of significant differences between subgroups, post-hoc analyses were based on the Tukey test $(p<0.05)$ or the Mann-Whitney $U$ test ( $p$ value obtained after applying Bonferroni correction, $p<0.05$; six possible comparisons).

\section{Results}

\section{Clinical response}

Out of a total of 45 female RA patients recruited for the study, 16 patients were excluded and the remaining 29 patients completed 15 months of treatment with TNF $\alpha$ I. In the excluded patients, TNF $\alpha$ I were discontinued due to the following reasons: no response in two patients, loss of response in three patients, intolerance in three patients, surgical procedures in four patients, and withdrawal of consent for participation in the therapy by four 
patients. Overall, 29 female RA patients who continued the TNF $\alpha$ I therapy for 15 months were included in our analysis and are presented in this study.

During the treatment with TNFaI, a significant clinical improvement in all RA patients was observed. Over the course of 3 months, 29 patients (100\%) qualified as good responders in accordance with the EULAR response criteria [26]. What is more, this effect was sustained up to the 15th month. Remission of RA occurred in $38 \%$ of patients at the 9th month and in $80 \%$ at the 15th month of treatment, while the remaining patients experienced low activity. The DAS28 score was significantly reduced 3,9 , and 15 months after the initiation of TNFaI therapy when compared with baseline. Furthermore, CRP and ESR levels decreased significantly after 9 and 15 months of treatment (Table 2).

\section{Plasma levels of total GAGs, KS, and HA}

The results regarding evaluation of plasma glycosaminoglycans (total GAGs, KS, and HA) were analyzed only in female RA patients who completed the whole 15-month TNF $\alpha$ I therapy $(n=29)$.

The concentrations of total plasma GAGs, KS, and $\mathrm{HA}$ in female RA patients before treatment with TNFoI and in healthy individuals are presented in Fig. 1a-c. Total GAGs and HA levels were significantly higher in RA women before anti-TNF- $\alpha$ therapy than in healthy subjects (both $p<0.001$; Fig. 1a, c). KS levels were not different in RA women before biological treatment in comparison to those of the controls ( $p=0.862$; Fig. 1b).

Three months after the initiation of TNFoI therapy, a statistically significant decrease in total GAG levels was observed in RA patients $(p<0.001$; Fig. 2a). Continued therapy resulted in a further decline $(p<0.001$; Fig. 2a), reaching total GAG levels characteristic of the agematched healthy controls after 15 months of treatment $(p=0.183$; Fig. 3a). Similarly, HA levels decreased significantly in response to anti-TNF- $\alpha$ therapy $(p<0.001$; Fig. 2c). Furthermore, anti-TNF- $\alpha$ treatment also led to normalization of HA in RA patients ( $p=0.826$; Fig. 3c). In contrast, KS levels in RA women were not affected by the treatment ( $p=0.744$; Fig. $2 b)$ and were not significantly different from those in healthy subjects after 15 months of TNF $\alpha$ I therapy ( $p=0.788$; Fig. 3b).

\section{Discussion}

TNF- $\alpha$ inhibitors provide a new standard in the treatment of RA. TNF $\alpha$ I administration for 15 months resulted in improvement in terms of disease activity, as shown by the decrease of DAS28 and CRP and the achievement of full remission or low disease activity in all RA patients. We also showed that this beneficial effect was associated with improvement in metabolism of ECM components, assessed through plasma sulfated and nonsulfated GAG levels in
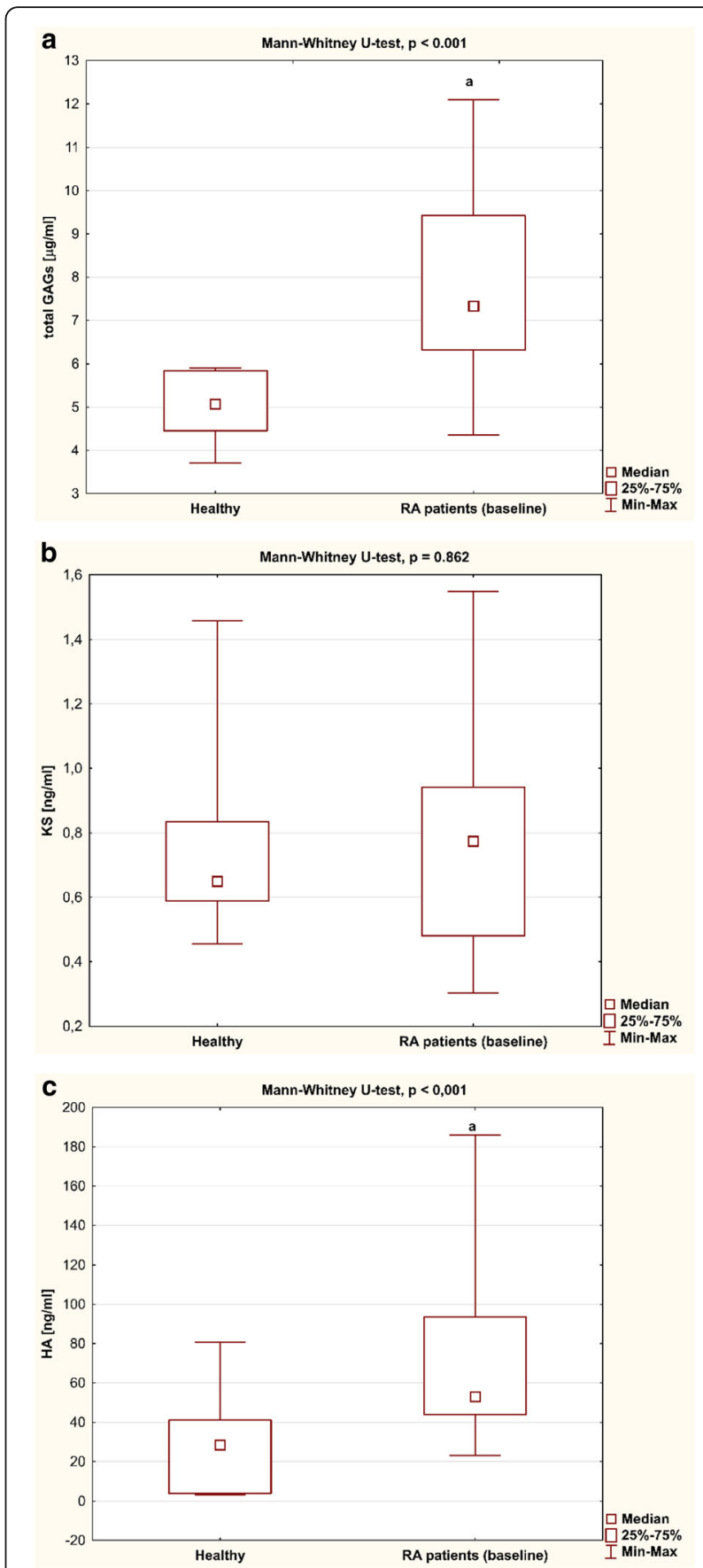

Fig. 1 Plasma levels of (a) GAGs, (b) KS, and (c) HA in RA patients $(n=29)$ before anti-TNF-a therapy and in healthy subjects $(n=20)$. Data analyzed using Mann-Whitney $U$ test. ${ }^{a} p<0.001$, compared to healthy subjects. GAG glycosaminoglycan, HA hyaluronic acid, KS keratan sulfate, Max maximum, Min minimum, RA rheumatoid arthritis

female RA patients. Indeed, a decrease in the total GAGs as well as HA levels toward normal values was observed under anti-TNF- $\alpha$ treatment. Only the plasma concentration of KS in RA women was not affected by the treatment. To the best of our knowledge, this is the first study that 

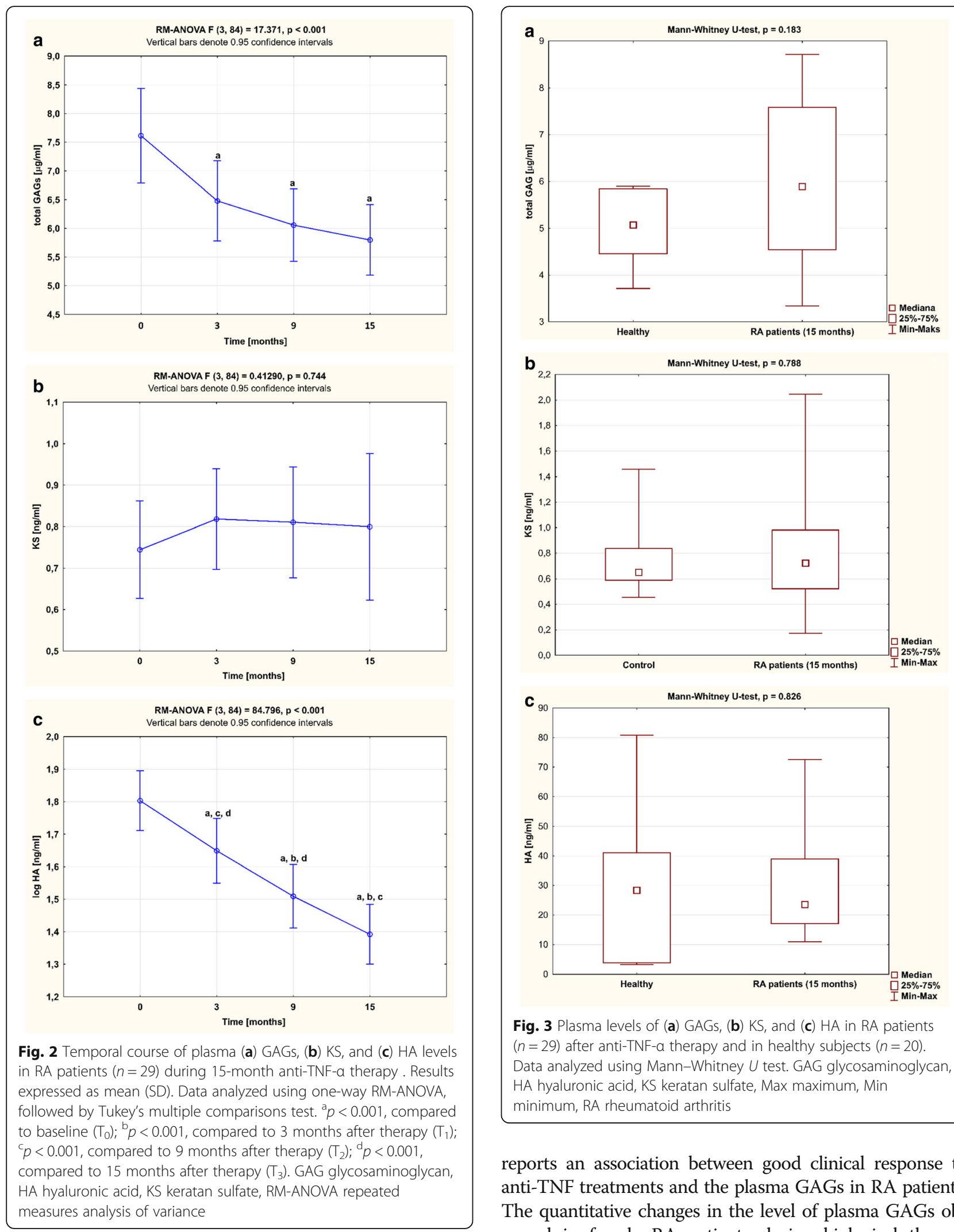

Fig. 3 Plasma levels of (a) GAGs, (b) KS, and (c) HA in RA patients $(n=29)$ after anti-TNF-a therapy and in healthy subjects $(n=20)$. Data analyzed using Mann-Whitney $U$ test. GAG glycosaminoglycan, HA hyaluronic acid, KS keratan sulfate, Max maximum, Min minimum, RA rheumatoid arthritis

reports an association between good clinical response to anti-TNF treatments and the plasma GAGs in RA patients. The quantitative changes in the level of plasma GAGs observed in female RA patients during biological therapy seem to result from an effective inhibition of the key proinflammatory cytokine, TNF- $\alpha$, rather than from direct 
impact of TNF $\alpha$ I on the tissue ECM remodeling. This suggestion is supported by the results of Jura-Półtorak et al. [15], who evaluated the plasma GAG profile in relation to disease activity in RA patients treated with conventional synthetic disease-modifying anti-rheumatic drugs (i.e., methotrexate or sulfasalazine). Similarly to our results, they demonstrated a significant increase in the total plasma GAG level in RA patients with a high disease activity (DAS28 > 5.1) in comparison to patients with a low disease activity (DAS28 $\leq 3.2$ ) [15]. Thus, the primary determinant of tissue PG/GAG turnover in RA patients is not the type of medication used for treatment of RA, but the control of disease activity. Moreover, consistent with our results, Jura-Półtorak et al. [15] found that the plasma level of GAGs was significantly elevated in RA patients with a high disease activity in comparison to healthy individuals. On the contrary, Friman et al. [14] showed that the plasma GAG concentrations in female patients with an active, erosive form of RA and in healthy subjects are similar. These discrepancies can result from methodological differences, especially with respect to the binding properties of a bed used in the ion exchange chromatography, that are crucial for the isolation of the plasma GAGs, which are characterized by huge heterogeneity in their electrical charge. In addition, the aforementioned divergences between our results and those of Friman et al. [14] might also be caused by differences in the disease activity of female RA patients, disease duration, as well as a type of anti-rheumatic drugs used. Summarizing, the blood accumulation of the total GAGs in the course of RA observed by us may indicate an increased tissue ECM turnover that is dependent on the disease activity.

Considering our results, we can suppose that decreased levels of total plasma GAGs during 15 months of TNFoI treatment might be due to reduced enzymatic and nonenzymatic catabolic processes. Matrix metalloproteinases (MMPs) and the family of proteins termed a disintegrin and metalloproteinase with a thrombospondin motifs (ADAMTS), as well as reactive oxygen species (ROS), participate in the turnover of tissue PGs/GAGs [9, 27-29]. With regard to the enzymes discussed earlier, the levels of MMP-3 and MMP-1 in serum of RA patients were found to be downregulated by TNF $\alpha$ I therapy [30-33]. Little is known about the effect of TNF- $\alpha$ inhibitors on ADAMTS activity in RA patients. However, inhibition of radiographic progression by TNF $\alpha$ I has been extensively documented through many clinical trials and it can be assumed that this beneficial result is at least partially related to the decreased activity of aggrecanase-1 (ADAMTS-4) and aggrecanase-2 (ADAMTS-5), proteinases responsible for cleavage of the main cartilage matrix PG, aggrecan [28, 34]. Another mechanism of alterations to the plasma GAG level in RA women may be connected with the ability of TNFaI to promote programmed cell death through the inhibition of nuclear factor- $\mathrm{kB}$ (NF- $\mathrm{kB}$ ) activation. As reported previously, etanercept as well as infliximab induce apoptosis of synovial fluid monocytes/macrophages, which are a constant source of MMPs and ADAMTS [28, 35]. Furthermore, TNF- $\alpha$ blockade normalizes the number of circulating monocytes in RA patients [36].

Besides the proteolytic breakdown of the ECM, oxidative damage plays a very prominent role in postsynthetic modifications of matrix components [29]. It is well known that excessive ROS formation in RA patients leads to peroxidation of the core proteins of PGs as well as the partial cleavage of GAG chains, thereby increasing the plasma GAG content $[29,37]$. Thus, it seems that normalization of the total plasma GAG level in RA women found in our study should be partly a reflection of the decreased nonenzymatic free radical degradation of these glycans. Indeed, anti-TNF- $\alpha$ therapy has been shown to suppress ROS generation in RA patients. It is reported that TNF- $\alpha$ blocking drugs reduce serum levels of reactive oxygen metabolites (ROM) as well as serum or urine levels of oxygen stress markers, including pentosidine, 8-hydroxy-2'-dexoyguanosine (8-OHdG), and $N^{\varepsilon}$-hexanoyl lysine ( ${ }^{\varepsilon}$-HEL) [38-40]. Inhibition of neutrophil migration into inflamed joints after initiation of anti-TNF- $\alpha$ treatment in RA patients has also been described [41]. The mentioned cells are abundant in RA synovial fluid, and their release of oxygen-derived free radicals and other inflammatory mediators may intensify the catabolism of PGs/GAGs [41].

Because most GAG types are also found in noncartilaginous tissues in significant amounts, their plasma levels do not essentially reflect cartilage PG catabolic activity in RA patients [42]. Keratan sulfate is an exception, in that more than $95 \%$ of KS is found in the aggrecan, a large aggregating PG of hyaline, elastic, and fibrous cartilages [42]. Thus, this may indicate that most plasma KS should come from cartilage PG degradation. Elevated levels of plasma KS have been observed in patients with osteoarthritis, as well as in healthy individuals characterized by higher sports activity $[43,44]$. In previous studies, the plasma concentrations of KS in RA patients have been shown to increase or decrease [15, 45-47]. In the present study, no significant difference was found in plasma KS levels between female RA patients and controls. We also showed that KS levels in RA women remained unchanged during 15 months of anti-TNF- $\alpha$ therapy. Our findings differ from those of Niki et al. [30], who demonstrated that the infliximab therapy-triggered increase of serum KS levels was more significant in the established RA patients than in the group of early RA patients. The possible explanation for these discrepancies could be connected with methodological differences in measurement of KS. In addition, these divergences might be caused in part by differences in 
gender of the patients, in the disease activity, and in amount of habitual exercise. In summary, the data presented here suggest that the potential usefulness of plasma antigen KS as an indicator of altered metabolic processes occurring in cartilaginous structures of RA patients during TNF $\alpha$ I therapy needs to be reevaluated.

In contrast to KS, plasma levels of the nonsulfated GAG hyaluronan could be helpful in predicting the efficacy of anti-TNF- $\alpha$ therapy in RA. The circulating levels of HA were greater in female RA patients before anti-TNF- $\alpha$ treatment when compared with age-matched healthy individuals. Similar findings have been reported in previous studies, none of which determined the gender of the patients $[15,48,49]$. In addition, TNFoI therapy led to a significant decrease in HA level, down to the values observed in healthy subjects. The outcomes of this study correspond with the results of Niki et al. [30]. They demonstrated that in the early RA group the serum level of HA gradually decreased during the 54-week infliximab therapy. Moreover, Niki et al. [30] found a strong linear correlation between the blood HA level in patients with early RA and DAS28, as well as inflammatory markers, such as CRP. These results may indicate that HA is an active participant in the inflammatory process accompanying RA. In the systemic circulation and in the synovial fluid of RA patients, the presence of low molecular weight fragments of hyaluronan (LMW-HA) was revealed. Such LMW-HA resulting from tissue HA depolymerization elicit proinflammatory responses by modulating toll-like receptor- 4 or by activating NF- $\kappa B$. NF- $\kappa B$ allows a vicious circle of chronic inflammation in RA by stimulating the expression of several proinflammatory cytokines such as interleukin (IL)-1, IL-6, and TNF- $\alpha$, which in turn induce alterations in HA metabolism $[8,9,12,29,50]$. Since hyaluronan is the most susceptible among all of the GAG types to degradation in the presence of ROS, it may be assumed that suppression of free radical-mediated HA fragmentation may be the main cause of normalization of plasma HA in the course of biological therapy that was observed in our study.

\section{Conclusions}

In summary, the results of our study have shown for the first time that anti-TNF- $\alpha$ therapy, which contributes to a clinical improvement in female RA patients, also has a beneficial effect on metabolism of tissue PGs/GAGs. Normalization of plasma levels of total GAGs and HA in RA patients suggests improvement of the ECM remodeling balance, mainly due to a decrease in PG/GAG breakdown. Our observations provide an additional mechanism for explaining cartilage-protective effects associated with anti-TNF- $\alpha$ treatment.

\section{Abbreviations}

8-OHdG: 8-Hydroxy-2'-dexoyguanosine; ACR: American College of Rheumatology; ADAMTS: Disintegrin and metalloproteinase with a thrombospondin motifs; BMI: Body mass index; CRP: C-reactive protein; CS/ DS: Chondroitin/dermatan sulfate; DAS28: Disease Activity Score based on the evaluation of 28 joints; ECM: Extracellular matrix; ELISA: Enzyme-linked immunosorbent assay; ESR: Erythrocyte sedimentation rate; EULAR: European League Against Rheumatism; GAG: Glycosaminoglycan; HA: Hyaluronic acid; HABP: Hyaluronic acid binding protein; HS/H: Heparan sulfate/heparin; IL: Interleukin; KS: Keratan sulfate; LMW-HA: Low molecular weight fragments of hyaluronan; MMP: Matrix metalloproteinase; NF-KB: nuclear factor-KB; $\mathrm{N}^{\varepsilon}$ HEL: NE-hexanoyl lysine; PG: Proteoglycan; RA: Rheumatoid arthritis; RF: Rheumatoid factor; RM-ANOVA: Repeated-measures analysis of variance; ROM: Reactive oxygen metabolites; ROS: Reactive oxygen species;

SJC28: Swollen joint count of 28 joints; TJC28: Tender joint count of 28 joints; TNF-a: Tumor necrosis factor alpha; TNFal: Tumor necrosis factor-alpha inhibitors; VAS: Visual analog scale

\section{Funding}

This study was funded by the National Science Centre, Poland (Grant No. 2013/09/N/NZ5/00815).

\section{Authors' contributions}

AS and AJ-P were responsible for study conception and design, performing the experiments, acquisition of data, statistical analysis and interpretation of data, writing and revising the article, and final approval of the version of the article to be published. EMK and AG were responsible for study conception and design, performing the experiments, revising the article, and final approval of the version of the article to be published. EJK and MK-M were responsible for study conception and design, including patient recruitment, data collection, and revising the article. KO was responsible for study conception and design, interpretation of data, writing and revising the article, and final approval of the version of the article to be published. All authors read and approved the final manuscript.

\section{Ethics approval and consent to participate}

During the entire investigation period we followed the guidelines and regulations of the Helsinki Declaration in 1975, as revised in 1983. The Ethical Committee of the Medical University of Silesia in Katowice approved the research protocol used in this study. All healthy volunteers and RA patients provided written informed consent.

\section{Consent for publication}

Not applicable.

\section{Competing interests}

The authors declare that they have no competing interests.

\section{Publisher's Note}

Springer Nature remains neutral with regard to jurisdictional claims in published maps and institutional affiliations.

\section{Author details}

${ }^{1}$ Department of Clinical Chemistry and Laboratory Diagnostics, School of Pharmacy with the Division of Laboratory Medicine in Sosnowiec, Medical University of Silesia in Katowice, Jedności 8, 41-200 Sosnowiec, Poland. ${ }^{2}$ Department of Internal Medicine and Rheumatology, School of Medicine in Katowice, Medical University of Silesia in Katowice, Ziołowa 45/47, 40-635 Katowice, Poland.

Received: 6 March 2018 Accepted: 3 September 2018

Published online: 18 September 2018

References

1. Smolen JS, Aletaha D, McInnes IB. Rheumatoid arthritis. Lancet. 2016;388: 2023-38.

2. Lauper K, Gabay C. Cardiovascular risk in patients with rheumatoid arthritis. Semin Immunopathol. 2017;39:447-59.

3. Song X, Lin Q. Genomics, transcriptomics and proteomics to elucidate the pathogenesis of rheumatoid arthritis. Rheumatol Int. 2017;37:1257-65.

4. Alam J, Jantan I, Bukhari SNA. Rheumatoid arthritis: recent advances on its etiology, role of cytokines and pharmacotherapy. Biomed Pharmacother. 2017;92:615-33. 
5. Angelotti F, Parma A, Cafaro G, Capecchi R, Alunno A, Puxeddu I. One year in review 2017: pathogenesis of rheumatoid arthritis. Clin Exp Rheumatol. 2017;35:368-78.

6. Brzustewicz E, Bryl E. The role of cytokines in the pathogenesis of rheumatoid arthritis - practical and potential application of cytokines as biomarkers and targets of personalized therapy. Cytokine. 2015;76:527-36.

7. Mateen S, Zafar A, Moin S, Khan AQ, Zubair S. Understanding the role of cytokines in the pathogenesis of rheumatoid arthritis. Clin Chim Acta. 2016:455:161-71.

8. Sorokin L. The impact of the extracellular matrix on inflammation. Nat Rev Immunol. 2010;10:712-23.

9. Bonnans C, Chou J, Werb Z. Remodelling the extracellular matrix in development and disease. Nat Rev Mol Cell Biol. 2014;15:786-801.

10. Soares da Costa D, Reis RL, Pashkuleva I. Sulfation of glycosaminoglycans and its implications in human health and disorders. Annu Rev Biomed Eng. 2017;19:1-26.

11. Mikami T, Kitagawa $H$. Sulfated glycosaminoglycans: their distinct roles in stem cell biology. Glycoconj J. 2017;34:725-35.

12. Volpi N, Schiller J, Stern R, Soltés L. Role, metabolism, chemical modifications and applications of hyaluronan. Curr Med Chem. 2009;16: 1718-45.

13. Gandhi NS, Mancera RL. The structure of glycosaminoglycans and their interactions with proteins. Chem Biol Drug Des. 2008;72:455-82.

14. Friman C, Juvani M, Skrifvars B. Acid glycosaminoglycans in plasma. II. Findings in rheumatoid arthritis. Scand J Rheumatol. 1977:6:177-82.

15. Jura-Półtorak A, Komosinska-Vassev K, Kotulska A, Kucharz EJ, Klimek K Kopec-Medrek M, et al. Alterations of plasma glycosaminoglycan profile in patients with rheumatoid arthritis in relation to disease activity. Clin Chim Acta. 2014;433:20-7.

16. Smolen JS, Landewé R, Bijlsma J, Burmester G, Chatzidionysiou K, Dougados $M$, et al. EULAR recommendations for the management of rheumatoid arthritis with synthetic and biological disease-modifying antirheumatic drugs: 2016 update. Ann Rheum Dis. 2017;76:960-77

17. Nam JL, Takase-Minegishi K, Ramiro S, Chatzidionysiou K, Smolen JS, van der Heijde D, et al. Efficacy of biological disease-modifying antirheumatic drugs: a systematic literature review informing the 2016 update of the EULAR recommendations for the management of rheumatoid arthritis. Ann Rheum Dis. 2017:76:1113-36.

18. Szeremeta A, Olczyk K. Tumor necrosis factor a antagonists in the treatment of the patients with rheumatoid arthritis. Reumatologia. 2012;50:438-43.

19. Arnett FC, Edworthy SM, Bloch DA, McShane DJ, Fries JF, Cooper NS, et al. The American Rheumatism Association 1987 revised criteria for the classification of rheumatoid arthritis. Arthritis Rheum. 1988;31:315-24.

20. Aletaha D, Neogi T, Silman AJ, Funovits J, Felson DT, Bingham CO, et al. 2010 rheumatoid arthritis classification criteria: an American College of Rheumatology/European League Against Rheumatism collaborative initiative. Ann Rheum Dis. 2010;69:1580-8.

21. Lu H, McDowell LM, Studelska DR, Zhang L. Glycosaminoglycans in human and bovine serum: detection of twenty-four heparan sulfate and chondroitin sulfate motifs including a novel sialic acid-modified chondroitin sulfate linkage hexasaccharide. Glycobiol Insights. 2010;2010:13-28.

22. Capobianco G, de Muro P, Cherchi GM, Formato M, Lepedda AJ, Cigliano A, et al. Plasma levels of C-reactive protein, leptin and glycosaminoglycans during spontaneous menstrual cycle: differences between ovulatory and anovulatory cycles. Arch Gynecol Obstet. 2010;282:207-13.

23. Volpi N, Galeotti F, Yang B, Linhardt RJ. Analysis of glycosaminoglycanderived, precolumn, 2-aminoacridone-labeled disaccharides with LCfluorescence and LC-MS detection. Nat Protoc. 2014;9:541-58.

24. Filisetti-Cozzi TM, Carpita NC. Measurement of uronic acids without interference from neutral sugars. Anal Biochem. 1991;197:157-62.

25. van den Hoogen BM, van Weeren PR, Lopes-Cardozo M, van Golde LM, Barneveld $\mathrm{A}$, van de Lest $\mathrm{CH}$. A microtiter plate assay for the determination of uronic acids. Anal Biochem. 1998;257:107-11.

26. Fransen J, van Riel PL. The disease activity score and the EULAR response criteria. Rheum Dis Clin N Am. 2009;35:745-57

27. Araki Y, Mimura T. Matrix metalloproteinase gene activation resulting from disordered epigenetic mechanisms in rheumatoid arthritis. Int J Mol Sci. 2017:18. https://doi.org/10.3390/ijms18050905.

28. García-Hernández MH, González-Amaro R, Portales-Pérez DP. Specific therapy to regulate inflammation in rheumatoid arthritis: molecular aspects. Immunotherapy. 2014;6:623-36.
29. Fuchs B, Schiller J. Glycosaminoglycan degradation by selected reactive oxygen species. Antioxid Redox Signal. 2014;21:1044-62.

30. Niki Y, Takeuchi T, Nakayama M, Nagasawa H, Kurasawa T, Yamada H, et al. Clinical significance of cartilage biomarkers for monitoring structural joint damage in rheumatoid arthritis patients treated with anti-TNF therapy. PLoS One. 2012;7:e37447. https://doi.org/10.1371/journal.pone.0037447.

31. Catrina Al, Lampa J, Ernestam S, af Klint E, Bratt J, Klareskog L, et al. Antitumour necrosis factor (TNF)-alpha therapy (etanercept) down-regulates serum matrix metalloproteinase (MMP)-3 and MMP-1 in rheumatoid arthritis. Rheumatology (Oxford). 2002;41:484-9.

32. den Broeder AA, Joosten LA, Saxne T, Heinegård D, Fenner $H$, Miltenburg AM, et al. Long term anti-tumor necrosis factor a monotherapy in rheumatoid arthritis: effect on radiological course and prognostic value of markers of cartilage turnover and endothelial activation. Ann Rheum Diss. 2002;61:311-8.

33. Kawashiri SY, Kawakami A, Ueki Y, Imazato T, Iwamoto N, Fujikawa K, et al. Decrement of serum cartilage oligomeric matrix protein (COMP) in rheumatoid arthritis (RA) patients achieving remission after 6 months of etanercept treatment: comparison with CRP, IgM-RF, MMP-3 and anti-CCP ab. Joint Bone Spine. 2010;77:418-20.

34. Dancevic CM, McCulloch DR. Current and emerging therapeutic strategies for preventing inflammation and aggrecanase-mediated cartilage destruction in arthritis. Arthritis Res Ther. 2014;16:429. https://doi.org/10. 1186/s13075-014-0429-9.

35. Catrina Al, Trollmo C, af Klint E, Engstrom M, Lampa J, Hermansson $Y$, et al. Evidence that anti-tumor necrosis factor therapy with both etanercept and infliximab induces apoptosis in macrophages, but not lymphocytes, in rheumatoid arthritis joints: extended report. Arthritis Rheum. 2005;52:61-72.

36. Chara L, Sánchez-Atrio A, Pérez A, Cuende E, Albarrán F, Turrión A, et al. Monocyte populations as markers of response to adalimumab plus MTX in rheumatoid arthritis. Arthritis Res Ther. 2012;14:R175. https://doi. org/10.1186/ar3928.

37. Mateen S, Moin S, Khan AQ, Zafar A, Fatima N. Increased reactive oxygen species formation and oxidative stress in rheumatoid arthritis. PLoS One. 2016;11:e0152925. https://doi.org/10.1371/journal.pone.0152925.

38. Hirao M, Yamasaki N, Oze H, Ebina K, Nampei A, Kawato $Y$, et al. Serum level of oxidative stress marker is dramatically low in patients with rheumatoid arthritis treated with tocilizumab. Rheumatol Int. 2012;32:4041-5.

39. Nakajima A, Aoki Y, Sonobe M, Takahashi H, Saito M, Nakagawa K. Serum level of reactive oxygen metabolites (ROM) at 12 weeks of treatment with biologic agents for rheumatoid arthritis is a novel predictor for 52-week remission. Clin Rheumatol. 2017;36:309-15.

40. Kageyama Y, Takahashi M, Nagafusa T, Torikai E, Nagano A. Etanercept reduces the oxidative stress marker levels in patients with rheumatoid arthritis. Rheumatol Int. 2008;28:245-51.

41. den Broeder AA, Wanten GJ, Oyen WJ, Naber T, van Riel PL, Barrera P. Neutrophil migration and production of reactive oxygen species during treatment with a fully human anti-tumor necrosis factor-alpha monoclonal antibody in patients with rheumatoid arthritis. J Rheumatol. 2003;30:232-7.

42. Caterson B, Melrose J. Keratan sulphate, a complex glycosaminoglycan with unique functional capability. Glycobiology 2018 published on 11 January 2018. doi: https://doi.org/10.1093/glycob/cwy003.

43. Woitge HW, Seibel MJ. Markers of bone and cartilage turnover. Exp Clin Endocrinol Diabetes. 2017;125:454-69.

44. Roos H, Dahlberg L, Hoerrner LA, Lark MW, Thonar EJ, Shinmei M, et al. Markers of cartilage matrix metabolism in human joint fluid and serum: the effect of exercise. Osteoarthr Cartil. 1995;3:7-14.

45. Haraoui B, Thonar EJ, Martel-Pelletier J, Goulet JR, Raynauld JP, Ouellet M, et al. Serum keratan sulfate levels in rheumatoid arthritis: inverse correlation with radiographic staging. J Rheumatol. 1994;21:813-7.

46. Poole AR, lonescu M, Swan A, Dieppe PA. Changes in cartilage metabolism in arthritis are reflected by altered serum and synovial fluid levels of the cartilage proteoglycan aggrecan. Implications for pathogenesis. J Clin Invest. 1994;94:25-33.

47. Spector TD, Woodward L, Hall GM, Hammond A, Williams A, Butler MG, et al. Keratan sulphate in rheumatoid arthritis, osteoarthritis, and inflammatory diseases. Ann Rheum Dis. 1992:51:1134-7.

48. Majeed M, McQueen F, Yeoman S, McLean L. Relationship between serum hyaluronic acid level and disease activity in early rheumatoid arthritis. Ann Rheum Dis. 2004;63:1166-8. 
49. Emlen W, Niebur J, Flanders G, Rutledge J. Measurement of serum hyaluronic acid in patients with rheumatoid arthritis: correlation with disease activity. J Rheumatol. 1996;23:974-8.

50. Sasaki Y, Uzuki M, Nohmi K, Kitagawa H, Kamataki A, Komagamine M, et al. Quantitative measurement of serum hyaluronic acid molecular weight in rheumatoid arthritis patients and the role of hyaluronidase. Int J Rheum Dis. 2011;14:313-9.

Ready to submit your research? Choose BMC and benefit from:

- fast, convenient online submission

- thorough peer review by experienced researchers in your field

- rapid publication on acceptance

- support for research data, including large and complex data types

- gold Open Access which fosters wider collaboration and increased citations

- maximum visibility for your research: over $100 \mathrm{M}$ website views per year

At BMC, research is always in progress.

Learn more biomedcentral.com/submissions 